\title{
Magnetic Properties of EuS-SrS Semiconductor Multilayer Structures
}

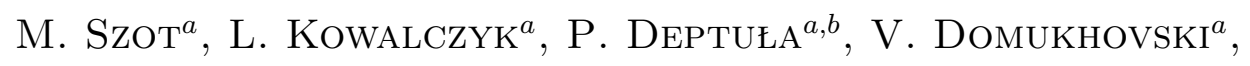 \\ V. Osinniy ${ }^{a}$, E. SmajeK ${ }^{a}$, A. Szczerbakow ${ }^{a}$, A.Yu. Sipatov ${ }^{c}$, \\ V.V. Volobuev ${ }^{c}$, A.G. Fedorov ${ }^{d}$ And T. Story ${ }^{a}$ \\ ${ }^{a}$ Institute of Physics, Polish Academy of Sciences \\ al. Lotników 32/46, 02-668 Warsaw, Poland \\ ${ }^{b}$ Department of Mathematics and Natural Sciences, College of Science \\ Cardinal S. Wyszyński University, Dewajtis 5, 01-185 Warsaw, Poland \\ ${ }^{c}$ National Technical University KPI \\ 21 Frunze Street, 61002 Kharkov, Ukraine \\ ${ }^{d}$ Institute for Scintillation Materials NASU \\ Lenin Ave. 60, 61001 Kharkov, Ukraine
}

\begin{abstract}
Magnetic and structural properties of EuS-SrS semiconductor multilayers were studied by SQUID and magneto-optical Kerr effect magnetometry techniques and by X-ray diffraction method. The multilayers composed of monocrystalline, lattice matched ferromagnetic EuS layers (thickness 35-50 $\AA$ ) and nonmagnetic SrS spacer layers (thickness 45-100 A) were grown epitaxially on $\mathrm{KCl}$ (001) substrates with $\mathrm{PbS}$ buffer layer. Ferromagnetic transition in EuS-SrS multilayers was found at the Curie temperature $T_{\mathrm{c}}=17 \mathrm{~K}$. The multilayers exhibit only weak in-plane magnetic anisotropy with [110] easy magnetization axis. Coercive field of EuS-SrS multilayers shows a linear increase with decreasing temperature. Magneto-optical mapping of magnetic hysteresis loops of the multilayers revealed good spatial homogeneity of their magnetic properties.
\end{abstract}

PACS numbers: 75.20.Ck, 75.30.Et

\section{Introduction}

In semiconductor EuS-SrS heterostructures, ferromagnetic layers of EuS are separated by diamagnetic SrS spacer layers. In previously studied EuS-PbS heterostructures, narrow band gap PbS spacer layers served as nonmagnetic quantum wells with ferromagnetic EuS barriers [1, 2]. In EuS-SrS structures the wide gap 
SrS layers are expected to form nonmagnetic electron barriers for ferromagnetic EuS quantum wells. As both EuS and SrS crystallize in the rock salt structure with their lattice parameters matching very well (mismatch of only $0.5 \%$ ), new high quality semiconductor ferromagnetic heterostructures can be epitaxially grown. EuS belongs to the family of europium chalcogenides and is a well-known Heisenberg nonmetallic ferromagnet with the lattice parameter $a_{0}=5.967 \AA$ and the energy gap of $1.65 \mathrm{eV}$. Ferromagnetic transition in EuS bulk crystals takes place at the Curie temperature $T_{\mathrm{c}}=16.5 \mathrm{~K}$. In thin epitaxial layers of EuS grown on $\mathrm{KCl}$ substrates the Curie temperature is influenced by the strain and size effects $[1,2]$. The thermal strain present in EuS layers due to the large difference in expansion coefficients of the layer and the $\mathrm{KCl}$ substrate leads to the in-plane biaxial compression of the EuS lattice. It results in an increase in the Curie temperature $T_{\mathrm{c}}$ by about $2 \mathrm{~K}$. For EuS layers thinner than about $30 \AA$ the Curie temperature is reduced due to the size effect which can be explained by a simple mean field model accounting for the reduced number of magnetic neighbors of Eu ions or by more elaborate theories developed for finite size ferromagnetic systems [1]. Strontium sulfide (SrS) is an insulating material with crystal lattice parameter $a_{0}=6.020 \AA$ and the energy gap about $7 \mathrm{eV}$. It serves as a nonmagnetic spacer layer material in the investigated EuS-SrS multilayer structures.

The aim of this work is to examine the basic magnetic and structural properties of EuS-SrS multilayers grown epitaxially on $\mathrm{KCl}$ (001) monocrystalline substrates. We consider the case of multilayers with relatively thick SrS nonmagnetic spacer ( $45 \AA$ and thicker) to deal with the system of ferromagnetic EuS layers magnetically decoupled from each other. The effect of antiferromagnetic interlayer coupling between EuS layers via nonmagnetic spacer, discovered in closely related in EuS-PbS multilayers with $\mathrm{PbS}$ spacer [3-6], was also found in EuS-SrS multilayers with ultrathin SrS spacer layer (below $10 \AA$ ). The EuS-SrS multilayers with ultrathin spacer exhibiting interlayer exchange effects will be discussed in other publication.

\section{Experimental}

EuS-SrS multilayers were grown by high vacuum deposition on freshly cleaved $\mathrm{KCl}$ (001) substrates with $\mathrm{PbS}$ buffer layer. Electron guns were used to evaporate EuS and SrS from solid state compound sources whereas for $\mathrm{PbS}$ a resistively heated tungsten boat was used. It was shown that various high crystal quality EuS-SrS multilayers (superlattices, trilayers, and bilayers) can be epitaxially grown by this technological method. The crystal quality of the layers was examined by X-ray diffraction method revealing, e.g. in EuS-SrS superlattices diffraction satellite peaks up to 3rd order. In EuS-SrS trilayers and bilayers studied in this work the layer thickness covered the range of 45-100 $\AA$ for nonmagnetic SrS spacer and 35-50 $\AA$ for ferromagnetic EuS layers. The results of the X-ray diffraction analysis of EuS-SrS-EuS trilayer structure presented in Fig. 1a clearly 
show a very good (001)-oriented monocrystalline structure of the layer. Despite a very small available volume of materials the standard X-ray experimental analysis shows diffraction peaks corresponding to each of the constituent layers and the substrate (Fig. 1b). The lattice parameters (along growth direction) of $\mathrm{PbS}$ buffer and EuS layers shown in Fig. 1b are larger than the reference values for bulk monocrystals. We assign this experimental finding to the effect of tensile deformation of the crystal lattice along the normal to the layer plane accompanying the biaxial compression due to thermal strain (quantified by the Poisson coefficient) [1].
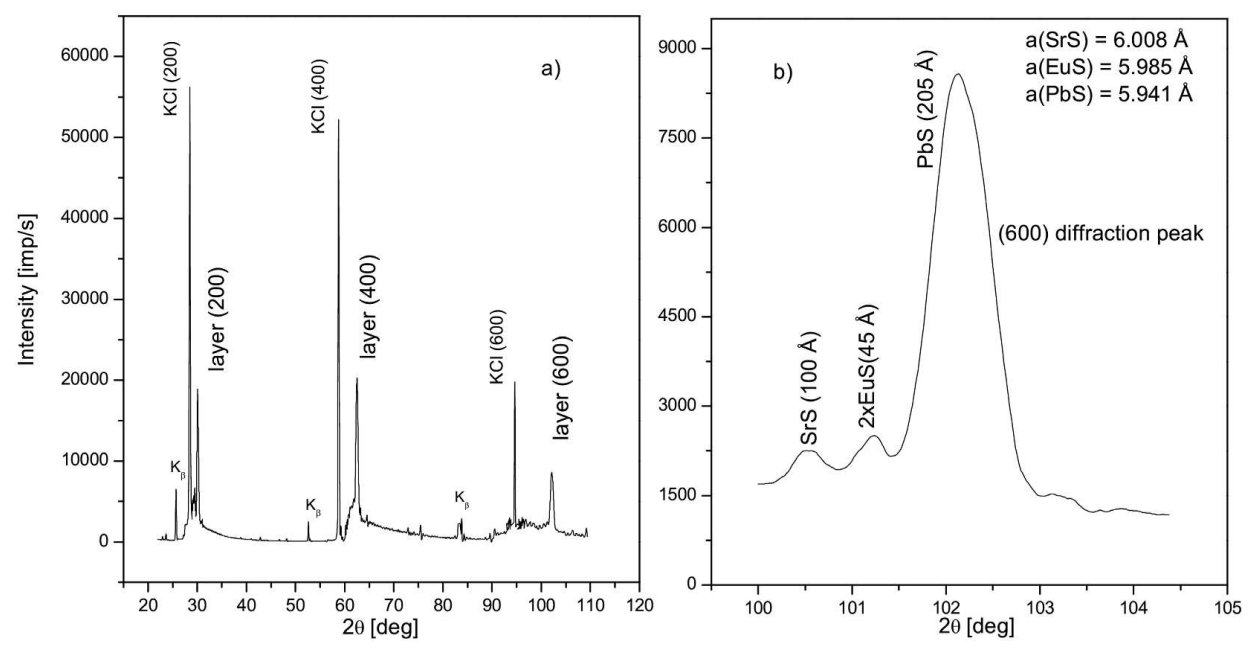

Fig. 1. X-ray diffraction $\theta-2 \theta$ analysis ( $\mathrm{Cu} K_{\alpha} \mathrm{X}$-ray line) of the crystal structure of $\operatorname{EuS}(45 \AA)-\operatorname{SrS}(100 \AA)-\operatorname{EuS}(45 \AA)-\operatorname{PbS}(205 \AA) / \mathrm{KCl}(001)$ multilayer (a). The small signal from the substrate due to $\mathrm{Cu} K_{\beta}$ X-ray line is also indicated. In (b) a detailed structure of (600) diffraction peak from the multilayer is presented with the identification of the rock salt lattice parameters of three constituent layers.

Magnetic properties of EuS-SrS multilayers were examined using superconductor SQUID magnetometry and magneto-optical Kerr effect (MOKE) magnetometry techniques in the temperature range $T=4-35 \mathrm{~K}$ and in external magnetic field $H \leq 300$ Oe applied in the (001) plane of the multilayer along [100] or [110] crystal directions. In the MOKE experiments, the linearly polarized light from $\mathrm{He}-\mathrm{Ne}$ laser was used in longitudinal MOKE geometry. The angle of incidence of light on the sample was about 30 degrees. The standard lock-in technique with photo-elastic modulator operating at $50 \mathrm{kHz}$ and a Si diode detector were used.

From the analysis of the temperature dependence of magnetization the ferromagnetic Curie temperature of EuS-SrS/KCl (001) multilayers was determined as equal to about $T_{\mathrm{c}}=17 \mathrm{~K}$. The sharp ferromagnetic transition in EuS-SrS-EuS trilayer is shown in Fig. 2a, where the magnetic hysteresis loops studied by SQUID 

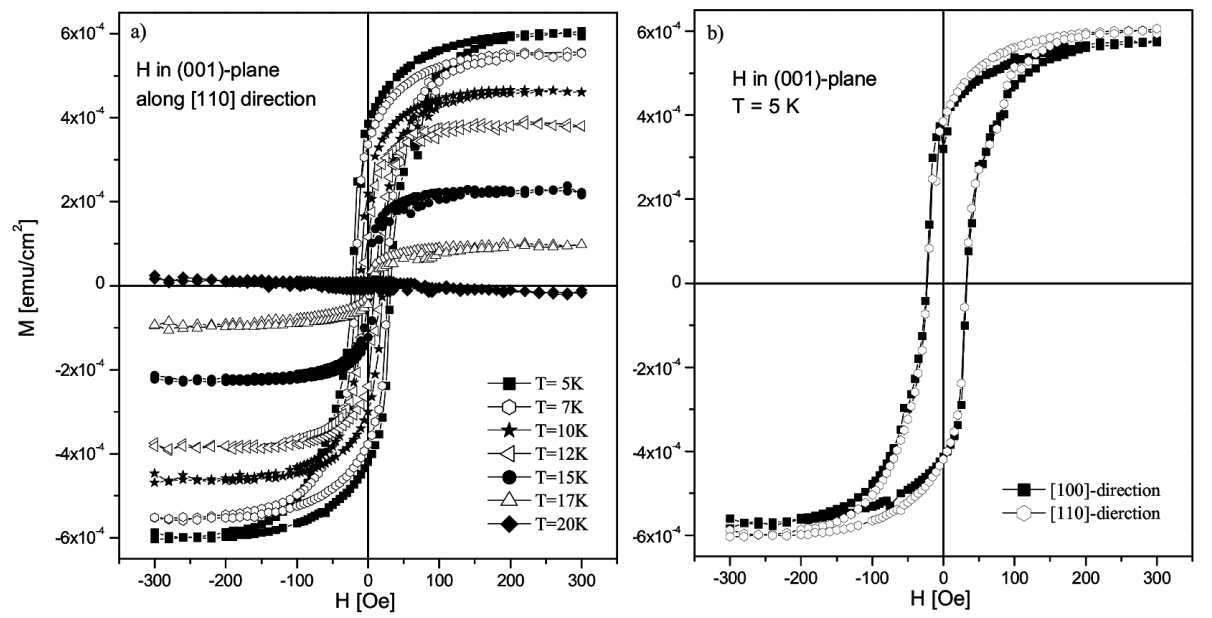

Fig. 2. (a) Magnetization loops (SQUID magnetometry) of $\operatorname{EuS}(45 \AA)-\operatorname{SrS}(50 \AA)-$ $\operatorname{EuS}(45 \AA)$ trilayer grown on $\mathrm{KCl}(001)$ substrate with $420 \AA$ thick PbS buffer layer. In (b) a very weak in-plane magnetic anisotropy observed in this multilayer is shown.
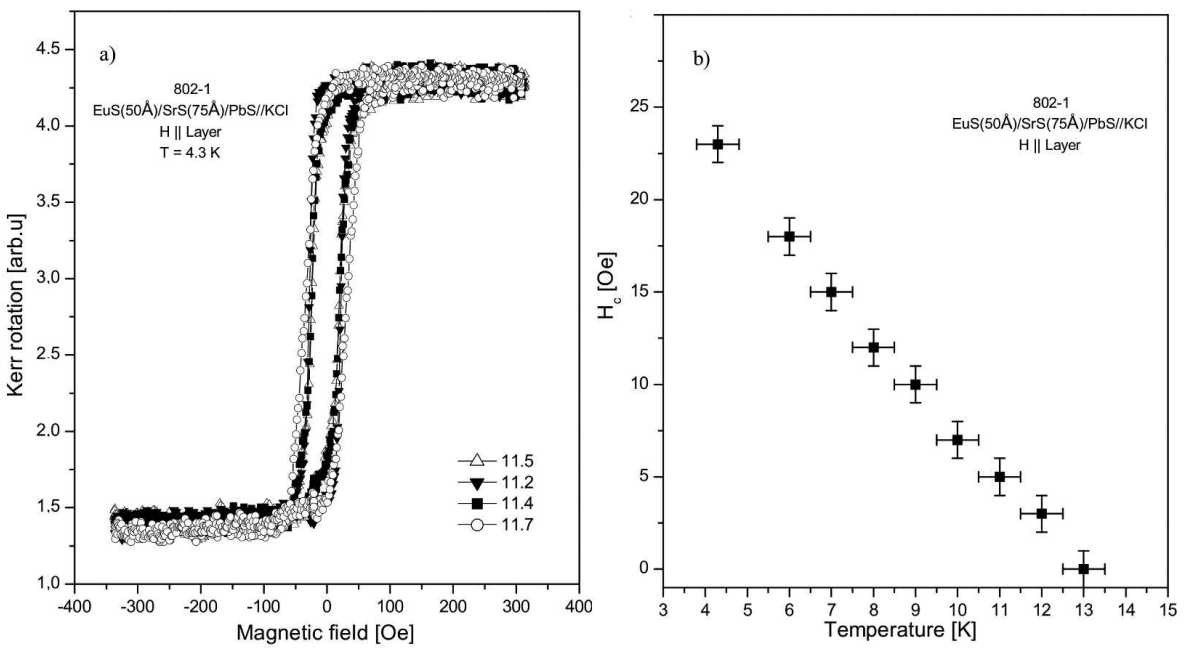

Fig. 3. (a) MOKE magnetometry analysis of magnetization loops in $\operatorname{EuS}(50 \AA)$ $\mathrm{SrS}(75 \AA)$ bilayer grown on $\mathrm{KCl}(001)$ substrate with $175 \AA$ thick $\mathrm{PbS}$ buffer layer. Different symbols refer to laser spot position (given in $\mathrm{cm}$ ) along the in-plane [100] crystal direction. In (b) the temperature dependence of the coercive field of this multilayer is presented.

magnetometry are presented in a broad temperature range. Magnetic hysteresis loops observed in EuS-SrS multilayers with $\mathrm{SrS}$ spacer thicker than about $10 \AA$ are of regular ferromagnetic character with typical values of key parameters at $5 \mathrm{~K}$ equal to 200 Oe for saturation field and 25 Oe for coercive field. 
The example of magnetic hysteresis loops observed for $\operatorname{EuS}(50 \AA)-\operatorname{SrS}(75 \AA)$ bilayer by the MOKE magnetometry technique is shown in Fig. 3a. To check the macroscopic magnetic homogeneity of the layers the laser spot of about $0.5 \mathrm{~mm}$ in diameter was scanned over $10 \times 3 \mathrm{~mm}^{2}$ area of the layer. In Fig. $3 \mathrm{~b}$ the temperature dependence of the coercive field measured in EuS-SrS bilayer by the MOKE magnetometry is also shown.

\section{Discussion and conclusions}

In $\mathrm{EuS}-\mathrm{SrS} / \mathrm{KCl}$ trilayers and bilayers studied in this work the well-defined ferromagnetic transition takes place at the Curie temperature of $T_{\mathrm{c}}=17 \mathrm{~K}$. It corresponds well to the ferromagnetic transition temperatures found in related EuS-PbS multilayers grown on $\mathrm{KCl}$ substrates [1]. The small increase in the Curie temperature for EuS-SrS/ $\mathrm{KCl}$ multilayers as compared to bulk EuS crystals is, most likely, caused by the in-plane compressive strain discussed above. This interpretation is supported by the X-ray diffraction observation that the EuS layer lattice parameter along the normal to the substrate plane is larger than in bulk EuS crystals. Also other basic ferromagnetic parameters of EuS-SrS multilayers, such as magnetic saturation field, coercive field, and in-plane magnetic anisotropy constant are typical of other EuS-based multilayers [4]. Due to very large demagnetization field $H_{\mathrm{D}}$ expected in EuS layers $\left(H_{\mathrm{D}}=4 \pi M \approx 15 \mathrm{kOe}\right.$ at $\left.T=0 \mathrm{~K}\right)$ the dipolar (shape) anisotropy is the dominant anisotropy mechanism in EuS-SrS multilayers with a negligible role of magnetocrystalline anisotropy.

In a series of EuS-SrS multilayers examined in this work we observed magnetization saturation values at $T=5 \mathrm{~K}$ in the range of $700-1000 \mathrm{emu} / \mathrm{cm}^{3}$. As the theoretical value for the ideal EuS layer (with $\mathrm{Eu}^{2+}$ ions possessing magnetic moment of 7 Bohr magnetons) is at $T=5 \mathrm{~K}$ equal to about $1100 \mathrm{emu} / \mathrm{cm}^{3}$, a small magnetization deficit in EuS layers is detected. This effect is usually associated with the presence of various crystal defects which promote the change of $\mathrm{Eu}^{2+}$ ions in $\mathrm{Eu}^{3+}$ (nonmagnetic) state. Since in EuS-SrS multilayers we deal with rather thin layers of 35-50 $\AA$, in which surface and interface morphology effects are unavoidable, we find the magnetization saturation values in EuS-SrS multilayers satisfactory. Also the MOKE magnetization mapping of the multilayers revealed their good magnetic homogeneity with some irregularities observed in magnetization hysteresis loops close to large morphological defects of the substrate (brought about by the cleaving process). We note that the comparison of magnetization loops obtained by SQUID and MOKE magnetometry techniques reveals somewhat more rectangular character of loops found by the MOKE technique. We expect that, in part, this effect is related to the 10 times smaller surface area probed by the MOKE $\left(1 \mathrm{~mm}^{2}\right)$ and by the SQUID $\left(10 \mathrm{~mm}^{2}\right)$ magnetometers.

In conclusion, we studied experimentally the magnetic properties and the crystal structure of new semiconductor multilayers composed of ferromagnetic layers of EuS and nonmagnetic SrS spacer layers. X-ray diffraction analysis confirmed 
a high crystal quality of (001)-oriented monocrystalline EuS-SrS layers grown on $\mathrm{KCl}$ substrates with the evidence for the presence of thermal in-plane compressive strain. The SQUID and MOKE magnetometry analysis of magnetization revealed a sharp ferromagnetic transition in EuS-SrS multilayers at $T_{\mathrm{c}}=17 \mathrm{~K}$, very weak in-plane magnetic anisotropy, and good macroscopic magnetic homogeneity of the layers.

\section{Acknowledgments}

The work of V.V. Volobuev was supported by Ukrainian President grant for young scientists research GP/F11/0010.

\section{References}

[1] A. Stachow-Wójcik, T. Story, W. Dobrowolski, M. Arciszewska, R.R. Gałązka, H.J.M. Swagten, W.J.M. de Jonge, A. Twardowski, A.Yu. Sipatov, Phys. Rev. B 60, 15220 (1999).

[2] T. Story, Phys. Status Solidi B 236, 310 (2003).

[3] H. Kȩpa, J. Kutner-Pielaszek, J. Blinowski, A. Twardowski, C.F. Majkrzak, T. Story, P. Kacman, R.R. Gałązka, K. Ha, H.J.M. Swagten, W.J.M. de Jonge, A.Yu. Sipatov, V.V. Volobuev, T.M. Giebultowicz, Europhys. Lett. 56, 54 (2001).

[4] C.J.P. Smits, A.T. Filip, H.J.M. Swagten, B. Koopmans, W.J.M. de Jonge, M. Chernyshova, L. Kowalczyk, K. Grasza, A. Szczerbakow, T. Story, W. Palosz, A.Yu. Sipatov, Phys. Rev. B 69, 224410 (2004).

[5] P. Sankowski, H. Kȩpa, P. Kacman, A.Yu. Sipatov, C.F. Majkrzak, T.M. Giebultowicz, Acta Phys. Pol. A 105, 607 (2004).

[6] L. Kowalczyk, S. Wrotek, P. Dziawa, V. Osinniy, M. Szot, T. Story, A.Yu. Sipatov, V.V. Volobuev, A.G. Fedorov, Acta Phys. Pol. A 110, 225 (2006). 